Review Article

\title{
Screening for Maternal Thyroid Dysfunction in Pregnancy: A Review of the Clinical Evidence and Current Guidelines
}

\author{
Donny L. F. Chang and Elizabeth N. Pearce \\ Section of Endocrinology, Diabetes and Nutrition, Boston University School of Medicine and Boston Medical Center, \\ 88 East Newton Street, Evans 201, Boston, MA 02118, USA
}

Correspondence should be addressed to Elizabeth N. Pearce; elizabeth.pearce@bmc.org

Received 12 December 2012; Accepted 22 April 2013

Academic Editor: Fereidoun Azizi

Copyright (c) 2013 D. L. F. Chang and E. N. Pearce. This is an open access article distributed under the Creative Commons Attribution License, which permits unrestricted use, distribution, and reproduction in any medium, provided the original work is properly cited.

\begin{abstract}
Observational studies have demonstrated that maternal thyroid dysfunction and thyroid autoimmunity in pregnancy may be associated with adverse obstetric and fetal outcomes. Treatment of overt maternal hyperthyroidism and overt hypothyroidism clearly improves outcomes. To date there is limited evidence that levothyroxine treatment of pregnant women with subclinical hypothyroidism, isolated hypothyroxinemia, or thyroid autoimmunity is beneficial. Therefore, there is ongoing debate regarding the need for universal screening for thyroid dysfunction during pregnancy. Current guidelines differ; some recommend an aggressive case-finding approach, whereas others advocate testing only symptomatic women or those with a personal history of thyroid disease or other associated medical conditions.
\end{abstract}

\section{Introduction}

It is well documented that maternal thyroid dysfunction is associated with adverse outcomes in the mother and fetus, including miscarriage, preterm delivery, eclampsia, pre-eclampsia, and placental abruption [1-6]. Decreased availability of maternal thyroid hormone may also impair neurological development of the fetus as several studies have reported decreased IQ in infants born to mothers with either overt hypothyroidism $(\mathrm{OH})$ [7], hypothyroxinemia [8], or thyroid peroxidase antibody (TPO Ab) positivity [9]. However, there is limited and conflicting evidence regarding the impact of intervention on improving health outcomes in pregnant women with subclinical hypothyroidism $(\mathrm{SH})$ and with euthyroid autoimmune disease in pregnancy.

There is ongoing debate regarding the need for universal screening for thyroid dysfunction during pregnancy. Current guidelines differ between an aggressive case finding approach [10-13] versus testing only symptomatic women or those with a personal history of thyroid disease or other associated medical condition [14, 15] (Box 1). The differing expert opinions are likely due to the fact that the majority of the evidence regarding thyroid dysfunction in pregnancy is based on observational studies. There is a paucity of large, prospective, randomized controlled trials demonstrating treatment benefits.

This review examines the current clinical evidence as it relates to screening for thyroid dysfunction and euthyroid autoimmune disease in pregnancy. We also compare the different management guidelines currently available, and how these guidelines have influenced clinical practice to date. We have referenced the current US guidelines along with The Cochrane Collaboration for this review. There may be other regional guidelines which we have not cited.

\section{Thyroid Function Testing in Pregnancy}

Normal pregnancy is associated with significant changes in maternal thyroid physiology. Serum thyroid-stimulating hormone (TSH) concentration is the initial and most reliable test for assessing thyroid function in pregnancy [16]. Serum TSH testing is relatively inexpensive, readily available, and is a reliable test in pregnancy, assuming that trimester-specific reference ranges are applied. A decline in TSH levels in the first trimester is seen due to elevation of human chorionic gonadotropin (hCG), which functions as a weak stimulator 
Joint Guidelines from the American Association of Clinical Endocrinologists and the American Thyroid Association (2012) [10]

Universal screening is not recommended for patients who are pregnant or are planning pregnancy, including assisted reproduction. Aggressive case finding, rather than universal screening, should be considered for patients who are planning pregnancy.

The Endocrine Society (2012) [11]

The committee could not reach agreement with regard to testing recommendations for all newly pregnant women, therefore 2 versions were presented.

(1) Some members recommended screening of all pregnant women for serum TSH abnormalities by the ninth week or at the time of their first visit.

(2) Some members recommended aggressive case finding to identify and test high-risk women (see Box 2) for elevated TSH concentrations by the ninth week or at the time of their first visit before or during pregnancy, and they recognize that in some situations ascertainment of the individual's risk status may not be feasible. In such cases, and where the local practice environment is appropriate, testing of all women by week 9 of pregnancy or at the first prenatal visit is reasonable (see Box 2). American Thyroid Association (2011) [12]

Universal screening of healthy women for thyroid dysfunction before pregnancy is not recommended. However, caregivers should identify individuals at high risk for thyroid illness (see Box 2) on the basis of their medical history, physical exam, or prior biochemical data.

The Cochrane Collaboration (2010) [13]

Until convincing data from randomised-controlled trials become available, targeted thyroid function testing in pregnancy should be implemented in women at risk for thyroid disease (e.g., pregestational diabetics), those with a family history of thyroid disease and symptomatic women. Consideration could be given to screening women with a personal history of preterm birth or recurrent miscarriage. At present, universal screening would not be based on firm evidence.

Society of Maternal-Fetal Medicine (2012) [14]

Current recommendations include thyroid testing for pregnant women "at risk," such as those with known thyroid disease, symptoms of overt thyroid disease, suspected goiter, and autoimmune medical disorders such as Type 1 diabetes mellitus.

American College of Obstetrics and Gynecology (2007) [15]

Based on current literature, thyroid testing in pregnancy should be performed on symptomatic women and those with a personal history of thyroid disease or other medical conditions associated with thyroid disease (e.g., diabetes mellitus). Without evidence that identification and treatment of pregnant women with subclinical hypothyroidism improves maternal or infant outcomes, routine screening for subclinical hypothyroidism currently is not recommended.

Box 1: Screening recommendations for thyroid disorders in pregnancy.

of the TSH receptor. Due to these dynamic changes during pregnancy, use of trimester-specific and assay-specific TSH normal ranges is recommended. Where such reference ranges are not available, the following cutoffs may be used: first trimester, $<2.5 \mathrm{mIU} / \mathrm{L}$; second trimester, $<3 \mathrm{mIU} / \mathrm{L}$; third trimester, $<3 \mathrm{mIU} / \mathrm{L}[11,12]$.

Thyroid function tests during pregnancy are also affected by estrogen-mediated increases in the level of thyroxinebinding globulins (TBG). Total T3 and T4 levels increase starting in early pregnancy, due to the increased TBG levels, so that the upper limit of normal for total T3 and T4 in pregnancy is approximately 1.5 -fold the upper limit of the nonpregnancy reference range. Free T4 assays may be unreliable in pregnancy due to interference by the high TBG levels [17, 18]. Method-specific and trimester-specific reference ranges for direct immunoassays of free $\mathrm{T} 4$ are not currently widely available. The free T4 index may be more reliable than free T4 assays during pregnancy, and its use is advocated by the 2012 Endocrine Society guidelines [11, 17].

\section{Hyperthyroidism}

Overt hyperthyroidism occurs in approximately $0.1-0.4 \%$ of pregnancies [19] and is defined as a serum TSH level below the trimester-specific reference range with elevated levels of free T3, free T4 or both. The most common cause of overt hyperthyroidism in pregnancy is Graves' disease. Other causes include gestational transient thyrotoxicosis, toxic adenoma or multinodular goiter, thyroiditis, or excessive hormone intake.

Definitive diagnosis can be more challenging in pregnancy since radioactive iodine thyroid scans are contraindicated. However, correlation with signs and symptoms may help elucidate the diagnosis. A diffuse goiter, ophthalmopathy, hyperthyroid symptoms prior to pregnancy, and serum thyroid hormone receptor antibody (TRAb) positivity favor the diagnosis of Graves' disease. Transient gestational thyrotoxicosis is more common in women with morning sickness, especially those with the most severe form, hyperemesis gravidarum [20].

Pregnant women with untreated overt hyperthyroidism are at increased risk for spontaneous miscarriage, congestive heart failure, thyroid storm, preterm birth, pre-eclampsia, fetal growth restriction, and increased perinatal morbidity and mortality [21-23]. Treatment of overt Graves' hyperthyroidism in pregnancy to achieve adequate metabolic control has been associated with improved pregnancy outcomes [24].

Subclinical hyperthyroidism is defined as a serum TSH level below the trimester-specific reference range with normal levels of free T3 and T4. Although various TSH cut-off values 
have been used in studies to define subclinical hyperthyroidism, in general, subclinical maternal hyperthyroidism has not been found to be associated with adverse maternal or fetal outcomes [5], and recommendations are for monitoring in pregnancy, but not therapy $[11,12]$.

\section{Overt Hypothyroidism}

Around $0.5 \%$ of all pregnant women will have overt hypothyroidism $(\mathrm{OH})[3,25]$, defined as an elevated TSH level with a decreased level of free T4 $[5,26,27]$. The most common etiology of $\mathrm{OH}$ in pregnant women is chronic autoimmune thyroiditis (Hashimoto's thyroiditis). Other causes of $\mathrm{OH}$ include endemic iodine deficiency (ID), and prior radioactive iodine therapy or thyroidectomy.

While severe endemic ID can lead to $\mathrm{OH}$, mild-tomoderate ID is more frequently associated with isolated hypothyroxinemia rather than $\mathrm{OH}$. Currently, 30 countries worldwide are considered iodine deficient [28]. Although median urinary iodine concentrations and other measures can be used to determine the iodine status of populations, there are no biomarkers to diagnose ID in individuals [29, 30].

Untreated $\mathrm{OH}$ in pregnancy has consistently been shown to be associated with an increased risk for adverse pregnancy complications, as well as detrimental effects on fetal neurocognitive development [7]. Specific adverse outcomes associated with maternal $\mathrm{OH}$ include increased risks for premature birth, low birth weight, and miscarriage [1]. Allan et al. and Abalovich et al. demonstrated an increased risk of fetal loss in patients with $\mathrm{OH}[3,4]$. Leung et al. demonstrated a $22 \%$ risk of gestational hypertension in pregnant women with $\mathrm{OH}$, higher than for euthyroid women or those with subclinical hypothyroidism (SH) [2].

Because of the clear associations between $\mathrm{OH}$ and risk to the mother and fetus, treatment of overt hypothyroidism during pregnancy is mandatory [10-12]. The goal of levothyroxine (LT4) treatment is to normalize maternal serum TSH values to within the trimester-specific pregnancy reference range [10-12].

\section{Subclinical Hypothyroidism (SH)}

SH is defined as an elevated TSH level with a normal level of circulating free $\mathrm{T} 4$. The prevalence of $\mathrm{SH}$ during pregnancy in the US is estimated to be $0.25-2.5 \%[3,25]$. Symptoms of $\mathrm{SH}$, if present, are typically subtle, and might be attributed to pregnancy. Even in $\mathrm{OH}$ individuals there can be a major discrepancy between symptoms and thyroid status. Canaris et al. demonstrated that although $\mathrm{OH}$ patients were more likely to report hypothyroid symptoms than euthyroid individuals, only $30 \%$ of $\mathrm{OH}$ patients were symptomatic whereas $17 \%$ of controls reported hypothyroid symptoms [31]. In addition, close to $20 \%$ of $\mathrm{OH}$ patients reported no symptoms at all. Thus, while the presence of symptoms may be suggestive of either $\mathrm{OH}$ or $\mathrm{SH}$, their absence fails to exclude it.

$\mathrm{SH}$ in pregnancy has been associated with adverse maternal outcomes in observational studies including eclampsia, pre-eclampsia, placental abnormalities, miscarriages, preterm labor, and low birth weights [1-5]. However, other studies have not found adverse obstetrical outcomes in subclinically hypothyroid pregnant women $[32,33]$ and adverse outcomes have varied from study to study.

A single prospective randomized control trial (RCT) to date has assessed the effect of LT4 therapy for mild maternal thyroid failure during pregnancy on offspring IQ [34]. Lazarus et al. randomized mildly hypothyroid or hypothyroxinemic pregnant women to LT4 treatment versus no treatment. At age 3, children of women treated with LT4 (started at a median gestational age of 13 weeks) had IQ tests which did not differ from the children of untreated women. These results have been criticized on the basis that intervention began in many women following the first trimester, which is the critical time for fetal brain development. Furthermore, IQ testing may not be the most sensitive method of assessing the effect of hypothyroidism on neural development in 3 year olds. Another ongoing large scale prospective randomized controlled trial sponsored by the National Institute of Child Health and Human Development is screening pregnant women less than 20 weeks gestation for $\mathrm{SH}$ or hypothyroxinemia, and randomizing to treatment with LT4 or placebo until delivery. The offspring will have annual developmental testing until age 5 to determine whether therapy is effective in improving IQ [35].

Treatment of $\mathrm{SH}$ is not universally advocated as there are limited data demonstrating a beneficial effect of thyroid hormone therapy on health outcomes [11, 12]. One RCT by Negro et al. has shown that treatment decreases the occurrence of adverse events in the mother and fetus [36]. The objective of the study was to determine if treatment of thyroid disease during pregnancy decreased the incidence of adverse outcomes and to compare the efficacy of universal screening versus case finding in detecting thyroid dysfunction. Whereas universal screening did not result in a decrease in adverse outcomes, treatment of thyroid abnormalities identified by screening a low-risk group resulted in a significant decrease in adverse outcomes.

Based on the Negro et al. study, the American Thyroid Association (ATA) recommended that women with SH who are TPO Ab positive should be treated with LT4 [12]. The ATA felt there was not enough evidence to recommend for or against LT4 treatment for TPO Ab negative women with $\mathrm{SH}$ and $\mathrm{TSH}<10 \mathrm{mIU} / \mathrm{L}$. However, the joint American Association of Clinical Endocrinologists (AACE)/ATA and The Endocrine Society (TES) guidelines recommend LT4 replacement in all women with SH $(\mathrm{TSH}>2.5 \mathrm{mIU} / \mathrm{L}$ in $1 \mathrm{st}$ trimester) regardless of TPO Ab status [10].

\section{Antithyroid Antibody Positivity in Pregnancy}

TPO and thyroglobulin (TG) autoantibodies can be detected in $10-20 \%$ of women of childbearing age [37]. The majority of women who test positive for thyroid autoantibodies are euthyroid. Sixteen percent of the women who are euthyroid and positive for TPO or TG antibodies in the first trimester will develop a TSH that exceeds $4.0 \mathrm{mIU} / \mathrm{L}$ 
by the third trimester [38], and 33\%-50\% of women who are positive for TPO or TG antibody in the first trimester will develop postpartum thyroiditis [39-41].

Thyroid autoimmunity in pregnancy has been associated with adverse pregnancy outcomes, including miscarriage [42-48], recurrent abortion [49-55], preterm births [37, 48, $56,57]$, and low IQ [8].

In a prospective study published in 1990 , the miscarriage rate was doubled in euthyroid women who tested positive for thyroid autoantibodies compared with women who tested negative [42]. The increased rate of miscarriage was not related to demographic variables nor to the presence of cardiolipin antibodies. A recent meta-analysis demonstrated an association between thyroid autoantibodies and spontaneous miscarriage, as well as preterm births [37]. A single RCT evaluated the effect of treatment of euthyroid TPO $\mathrm{Ab}$ positive pregnant women on miscarriage and showed a significant reduction in miscarriage rates, as well as the rates of preterm births [48]. There is currently an ongoing large, multicenter, double blind, placebo-controlled RCT in the United Kingdom to determine the efficacy of low dose LT4 treatment for obstetric and neonatal outcomes in women with thyroid antibodies (TABLET trial) [58].

The data for an association between thyroid antibodies and recurrent pregnancy loss are less robust than for sporadic loss and somewhat contradictory. Recurrent miscarriages can be due to many potential causes, and endocrine dysfunction may only account for $15 \%-20 \%$ of all cases. In addition, many of the previous trials did not control for other potential causes of recurrent losses. Therefore, none of the guidelines recommend universal TPO Ab testing or treatment of euthyroid $\mathrm{TPO} \mathrm{Ab}$ positive women [10-12].

\section{Isolated Hypothyroxinemia}

Isolated hypothyroxinemia is defined as a normal maternal TSH concentration with FT4 concentrations in the lower 5th or 10th percentiles of the reference range. Observational studies to date have not shown any adverse obstetric outcomes $[32,59]$. However, it is unclear whether isolated maternal hypothyroxinemia is associated with adverse neurodevelopmental outcomes. Pop et al. reported a decrease in psychomotor test scores among offspring born to women with FT4 indices in the lowest 10th percentile [60]. Henrichs et al. observed a similar reduction in the IQ of the offspring of mothers with isolated hypothyroxinemia during the first trimester [61]. Recent data from The Netherlands showed that children born to women with isolated hypothyroxinemia had a 1.5- to 2-fold increased risk of cognitive delays in early childhood [62]. The Controlled Antenatal Thyroid Study did not demonstrate a benefit of treatment in this subgroup; however, conclusions were limited due to the small numbers of women and the post-hoc nature of the analysis [34]. Other studies have not found a correlation between maternal thyroid hypofunction in pregnancy and offspring neurodevelopment [63-65].

Although there are limited data to suggest harm from isolated hypothyroxinemia, no interventional data to date has shown improvement with LT4 therapy. Thus, the ATA
2012 guidelines do not recommend routine screening or treatment for isolated hypothyroxinemia in pregnancy [12]. The Endocrine Society 2012 guidelines do not comment on isolated hypothyroxinemia in pregnancy [10].

\section{Screening for Thyroid Dysfunction}

Given that most thyroid dysfunction that occurs in pregnant women is $\mathrm{SH}$, and given the lack of clear data for efficacy of treatment, there is ongoing debate regarding the need for universal screening for thyroid dysfunction during pregnancyversus a case-finding approach [10-13] versus testing only symptomatic women or those with personal history of thyroid disease or other associated medical conditions $[14,15]$ (Box 1).

Appropriately dosed LT4 therapy in pregnancy does not confer any risks for the mother or fetus. However, the benefits of treating SH are unclear based on current published RCTs. The potential risks of universal screening would be: (1) costs of treatment, followup, and monitoring, (2) possible misinterpretation of TFTs resulting in inappropriate treatment, and (3) inappropriately dosed LT4 treatment for SH women, resulting in over- or undertreatment. Experienced caregivers should be involved in interpretation of TFTs in pregnancy to avoid misdiagnoses and initiation of inappropriate treatments (e.g., misinterpreting a low TSH as abnormal and inappropriately initiating treatment for hyperthyroidism). If patients are started on LT4 therapy, they need close monitoring to ensure euthyroidism. In a survey of a nonpregnant population, Canaris et al. demonstrated that over 40 percent of patients taking thyroid medications were not at target range and were either hypo- or hyperthyroid [66].

The most recent practice guidelines from the American College of Obstetricians and Gynecology (ACOG) in 2007 recommend thyroid testing only in high-risk pregnant women who are symptomatic, or have a personal history of thyroid disorders, personal history of type I diabetes or other autoimmune disorders [15]. The 2007 ACOG guidelines specifically do not recommend testing in asymptomatic women or women with small goiters. The Society for Maternal-Fetal Medicine supports the recommendations of ACOG [14].

The American Thyroid Association (ATA) in 2011 recommended against universal screening of healthy women for thyroid dysfunction during pregnancy [12]. However, a case-finding approach was advocated to identify individuals at high risk for hypothyroidism (see Box 2). In guidelines published in 2012, the Endocrine Society Task Force could not reach agreement on thyroid testing recommendations for pregnant women [11]. Some members recommended screening of all pregnant women for serum TSH abnormalities by the 9th week or at the time of their first visit. Others recommended against universal screening of pregnant women at the time of their first visit and instead supported aggressive case finding to identify high-risk women. This case-finding approach is similar to the 2011 ATA guidelines (see Box 2). The American Association of Clinical Endocrinologists (AACE)/ATA Hypothyroidism guidelines also currently recommend a case-finding approach [10]. 


\section{Efficacy of Case Finding}

Recent studies have examined the efficacy of the previous Endocrine Society 2007 guidelines' targeted case-finding approach in identifying women with thyroid dysfunction during early pregnancy [67]. Vaidya et al. showed that among a cohort of pregnant women, serum TSH levels were $>4.2 \mathrm{mIU} / \mathrm{L}$ in $2.6 \%$ of women and the prevalence was higher in the high-risk group versus the low-risk group [68]. However, $30 \%$ of the women with an elevated TSH were in the low-risk population, suggesting that the 2007 case-finding guidelines [67] would miss about one third of pregnant women with subclinical and overt hypothyroidism. In another study, Horacek et al. estimated that $55 \%$ of women with thyroid abnormalities (including positive thyroid antibody test results and hypothyroxinemia as well as subclinical and overt hypothyroidism) would have been missed using a case-finding approach rather than a universal screening approach [69]. In a retrospective cohort study performed in 2011, we reported that among 983 consecutive pregnant women in Boston, up to $80 \%$ of women with elevated TSH levels might have been missed using a case-finding approach rather than universal screening [70].

It is important to note that the previous studies were based on a slightly less comprehensive case-finding approach from the Endocrine Society 2007 [67] compared to the newer guidelines from the Endocrine Society in 2012 [11] and the ATA in 2011 [12]. Studies are needed to determine how effective the new case-finding criteria are at detecting overt and subclinical hypothyroidism in pregnancy.

\section{Thyroid Pregnancy Screening in Current Clinical Practice}

Conflicting guidelines can lead to variability in testing rates among practitioners and across regions [71, 72]. A retrospective national US study which included 502,036 pregnant women reported that $23 \%$ were tested for gestational hypothyroidism, of whom $15.5 \%$ had elevated serum TSH values [73]. In 2009, Haymart conducted a survey of Obstetricians and Family Medicine practitioners from Wisconsin and showed that only $11.5 \%$ of providers had read the Endocrine Society 2007 guidelines, but that reading the guidelines was associated with increased likelihood of screening for thyroid disease risk [72]. In 2010, Vaidya et al. surveyed members of the European Thyroid Association; 42\% of respondents reported screening all pregnant women for hypothyroidism, $43 \%$ reported that they used targeted case finding, and $17 \%$ of respondents did not perform routine thyroid testing [74]. Our 2011 Boston study at a single academic center showed that there was a high rate of screening (84.6\%) [70].

\section{Cost-Effectiveness Studies}

A recent study comparing ACOG 2002 guidelines (screening only symptomatic women or those with history of thyroid or associated diseases) versus universal screening for $\mathrm{SH}$ in pregnancy demonstrated that universal screening was by far the most cost-effective strategy under a wide range of
Targeted case finding criteria:

(1) Age $>30$ years

(2) Personal history of thyroid dysfunction

(3) Prior head or neck irradiation

(4) Prior thyroid surgery

(5) Family history

(6) Symptoms

(7) Presence of Goiter

(8) TPO Ab positivity

(9) Autoimmunity

(10) Infertility

(11) Miscarriage or preterm delivery

(12) Iodine deficient population

(13) Medications and iodinated contrast media*

(14) Morbid obesity $\left(\mathrm{BMI}>40 \mathrm{~kg} / \mathrm{m}^{2}\right)^{*}$

* Only for American Thyroid Association 2011 guidelines.

Box 2: Case-finding approach for high risk patients based on The Endocrine Society 2012 [11] and American Thyroid Association 2011 [12] guidelines.

circumstances [75]. Using a decision analysis model, Thung et al. determined that universally screening 100,000 women can result in over $\$ 8,000,000$ in cost savings and improve offspring outcome by reducing the number of offspring with cognitive impairment. The cost-savings were determined from the relatively low cost of thyroid screening tests and treatment of SH compared to the relatively large additional lifetime costs incurred by individuals with neurodevelopmental impairment. However, a benefit of treatment on offspring neurodevelopment has not to date been demonstrated in interventional studies [34].

Another recent study by Dosiou et al. also supports universal screening of pregnant women as a cost-effective measure in various clinical scenarios [76]. They developed a model in which women were screened in the first trimester of pregnancy with TSH and TPOA Ab. Women with TSH elevations underwent further testing, and treatment with LT4 was initiated when indicated. Their analysis showed that universal screening of pregnant women was a cost-effective measure compared to no screening at all, but also when compared to screening of high-risk women alone. Importantly, universal screening remained cost-effective even when only $\mathrm{OH}$, rather than $\mathrm{SH}$, was detected and treated.

\section{Conclusions}

The issue of universal screening for thyroid dysfunction and euthyroid autoimmune disease in pregnancy remains controversial. Observational studies have demonstrated adverse maternal and fetal outcomes in both women with subclinical hypothyroidism and in euthyroid women who test positive for thyroid autoantibodies. To date, there is limited evidence to demonstrate that LT4 treatment can improve outcomes.

In our personal practice, we perform screening TSH during pregnancy, and treat all pregnant patients with $\mathrm{SH}$. 
However, we cannot recommend universal screening at this time given the paucity of interventional studies.

As more data become available regarding the effectiveness of treatment and screening for thyroid dysfunction in pregnant women, recommendations for thyroid testing in pregnancy and clinical practice patterns will likely become more uniform.

\section{References}

[1] L. E. Davis, K. J. Leveno, and F. G. Cunningham, "Hypothyroidism complicating pregnancy," Obstetrics and Gynecology, vol. 72, no. 1, pp. 108-112, 1988.

[2] A. S. Leung, L. K. Millar, P. P. Koonings, M. Montoro, and J. H. Mestman, "Perinatal outcome in hypothyroid pregnancies," Obstetrics and Gynecology, vol. 81, no. 3, pp. 349-353, 1993.

[3] W. C. Allan, J. E. Haddow, G. E. Palomaki et al., "Maternal thyroid deficiency and pregnancy complications: implications for population screening," Journal of Medical Screening, vol. 7, no. 3, pp. 127-130, 2000.

[4] M. Abalovich, S. Gutierrez, G. Alcaraz, G. Maccallini, A. Garcia, and O. Levalle, "Overt and subclinical hypothyroidism complicating pregnancy," Thyroid, vol. 12, no. 1, pp. 63-68, 2002.

[5] B. M. Casey, J. S. Dashe, C. E. Wells et al., "Subclinical hypothyroidism and pregnancy outcomes," Obstetrics and Gynecology, vol. 105, no. 2, pp. 239-245, 2005.

[6] G. Ashoor, N. Maiz, M. Rotas, F. Jawdat, and K. H. Nicolaides, "Maternal thyroid function at 11 to 13 weeks of gestation and subsequent fetal death," Thyroid, vol. 20, no. 9, pp. 989-993, 2010.

[7] J. E. Haddow, G. E. Palomaki, W. C. Allan et al., "Maternal thyroid deficiency during pregnancy and subsequent neuropsychological development of the child," The New England Journal of Medicine, vol. 341, no. 8, pp. 549-555, 1999.

[8] V. J. Pop, J. L. Kuijpens, A. L. van Baar et al., "Low maternal free thyroxine concentrations during early pregnancy are associated with impaired psychomotor development in infancy," Clinical Endocrinology, vol. 50, no. 2, pp. 147-155, 1999.

[9] V. J. Pop, E. de Vries, A. L. van Baar et al., "Maternal thyroid peroxidase antibodies during pregnancy: a marker of impaired child development?" Journal of Clinical Endocrinology and Metabolism, vol. 80, no. 12, pp. 3561-3566, 1995.

[10] J. R. Garber, R. H. Cobin, H. Gharib et al., "Clinical practice guidelines for hypothyroidism in adults: co-sponsored by American Association of Clinical Endocrinologists and the American Thyroid Association," Endocrine Practice, vol. 11, pp. 1-207, 2012.

[11] L. De Groot, M. Abalovich, E. K. Alexander et al., "Management of thyroid dysfunction during pregnancy and postpartum: an Endocrine Society clinical practice guideline," Journal of Clinical Endocrinology and Metabolism, vol. 97, no. 8, pp. 25432565, 2012.

[12] A. Stagnaro-Green, M. Abalovich, E. Alexander et al., "Guidelines of the American Thyroid Association for the diagnosis and management of thyroid disease during pregnancy and postpartum," Thyroid, vol. 21, no. 10, 2011.

[13] S. M. Reid, P. Middleton, M. C. Cossich, and C. A. Crowther, "Interventions for clinical and subclinical hypothyroidism in pregnancy," Cochrane Database of Systematic Reviews, vol. 7, Article ID CD007752, 2010.
[14] Society for Maternal-Fetal Medicine (SMFM) and C. GyamfiBannerman, "Screening for thyroid disease during pregnancy," Contemporary OB/Gyn, vol. 57, no. 8, 2012, http://digital .healthcaregroup.advanstar.com/nxtbooks/advanstar/obgyn 201208/\#/8.

[15] Committee on Patient Safety and Quality Improvement and Committee on Professional Liability, "ACOG Committee Opinion No. 381: subclinical hypothyroidism in pregnancy," Obstetrics and Gynecology, vol. 110, no. 4, pp. 959-960, 2007.

[16] D. Glinoer and C. A. Spencer, "Serum TSH determinations in pregnancy: how, when and why?" Nature Reviews Endocrinology, vol. 6, no. 9, pp. 526-529, 2010.

[17] R. H. Lee, C. A. Spencer, J. H. Mestman et al., "Free T4 immunoassays are flawed during pregnancy," American Journal of Obstetrics and Gynecology, vol. 200, no. 3, pp. 260.e1-260.e6, 2009.

[18] R. Sapin, M. D'Herbomez, and J. L. Schlienger, "Free thyroxine measured with equilibrium dialysis and nine immunoassays decreases in late pregnancy," Clinical Laboratory, vol. 50, no. 910, pp. 581-584, 2004.

[19] D. Glinoer, “Thyroid hyperfunction during pregnancy," Thyroid, vol. 8, no. 9, pp. 859-864, 1998.

[20] T. M. Goodwin, M. Montoro, and J. H. Mestman, “Transient hyperthyroidism and hyperemesis gravidarum: clinical aspects," American Journal of Obstetrics and Gynecology, vol.167, no. 3, pp. 648-652, 1992.

[21] L. E. Davis, M. J. Lucas, G. D. V. Hankins, M. L. Roark, and F. G. Cunningham, "Thyrotoxicosis complicating pregnancy," American Journal of Obstetrics and Gynecology, vol. 160, no. 1, pp. 63-70, 1989.

[22] L. K. Millar, D. A. Wing, A. S. Leung, P. P. Koonings, M. N. Montoro, and J. H. Mestman, "Low birth weight and preeclampsia in pregnancies complicated by hyperthyroidism," Obstetrics and Gynecology, vol. 84, no. 6, pp. 946-949, 1994.

[23] A. Kriplani, K. Buckshee, V. L. Bhargava, D. Takker, and A. C. Ammini, "Maternal and perinatal outcome in thyrotoxicosis complicating," European Journal of Obstetrics Gynecology and Reproductive Biology, vol. 54, no. 3, pp. 159-163, 1994.

[24] N. Momotani, J. Noh, and H. Oyanagi, "Antithyroid drug therapy for Graves' disease during pregnancy: optimal regimen for fetal thyroid status," The New England Journal of Medicine, vol. 315, no. 1, pp. 24-28, 1986.

[25] R. Z. Klein, J. E. Haddow, J. D. Faix et al., "Prevalence of thyroid deficiency in pregnant women," Clinical Endocrinology, vol. 35, no. 1, pp. 41-46, 1991.

[26] A. Stagnaro-Green, S. H. Roman, R. H. Cobin, E. El-Harazy, S. Wallenstein, and T. F. Davies, "A prospective study of lymphocyte-initiated immunosuppression in normal pregnancy: evidence of a T-cell etiology for postpartum thyroid dysfunction," Journal of Clinical Endocrinology and Metabolism, vol. 74, no. 3, pp. 645-653, 1992.

[27] A. Stagnaro-Green, "Overt hyperthyroidism and hypothyroidism during pregnancy," Clinical Obstetrics and Gynecology, vol. 54, no. 3, pp. 478-487, 2011.

[28] E. N. Pearce, M. Andersson, and M. Zimmermann, "Global iodine nutrition-where do we stand in 2013?" Thyroid, vol. 23, no. 5, pp. 523-528, 2013.

[29] WHO, UNICEF, and ICCIDD, Assessment of Iodine Deficiency Disorders and Monitoring Their Elimination. A Guide for Programme Managers, WHO, Geneva, Switzerland, 3rd edition, 2007. 
[30] M. Andersson, V. Karumbunathan, and M. B. Zimmermann, "Global iodine status in 2011 and trends over the past decade," Journal of Nutrition, vol. 142, no. 4, pp. 744-750, 2012.

[31] G. J. Canaris, J. F. Steiner, and E. C. Ridgway, "Do traditional symptoms of hypothyroidism correlate with biochemical disease?" Journal of General Internal Medicine, vol. 12, no. 9, pp. 544-550, 1997.

[32] J. Cleary-Goldman, F. D. Malone, G. Lambert-Messerlian et al., "Maternal thyroid hypofunction and pregnancy outcome," Obstetrics and Gynecology, vol. 112, no. 1, pp. 85-92, 2008.

[33] T. Männistö, M. Vääräsmäki, A. Pouta et al., "Perinatal outcome of children born to mothers with thyroid dysfunction or antibodies: a prospective population-based cohort study," Journal of Clinical Endocrinology and Metabolism, vol. 94, no. 3, pp. 772779, 2009.

[34] L. H. Lazarus, J. P. Bestwick, S. Channon et al., "Antenatal thyroid screening and childhood cognitive function," The New England Journal of Medicine, vol. 366, no. 6, pp. 493-501, 2012.

[35] Eunice Kennedy Shriver National Institute of Child Health and Human Development (NICHD), "Thyroid Therapy for Mild Thyroid Deficiency in Pregnancy (TSH)," In, http://www .nhlbi.nih.gov/meetings/workshops/cardiorenal-hf-hd.htm, Bethesda (MD): National Library of Medicine (US). 2000, http: clinicaltrials.gov/ct2/show/NCT00388297, NLM Identifier: NCT00388297.

[36] R. Negro, A. Schwartz, R. Gismondi, A. Tinelli, T. Mangieri, and A. Stagnaro-Green, "Universal screening versus case finding for detection and treatment of thyroid hormonal dysfunction during pregnancy," Journal of Clinical Endocrinology and Metabolism, vol. 95, no. 4, pp. 1699-1707, 2010.

[37] S. Thangaratinam, A. Tan, E. Knox, M. D. Kilby, J. Franklyn, and A. Coomarasamy, "Association between thyroid autoantibodies and miscarriage and preterm birth: Meta-analysis of evidence," British Medical Journal, vol. 342, no. 7806, pp. 1-8, 2011.

[38] D. Glinoer, M. Riahi, J. P. Grun, and J. Kinthaert, "Risk of subclinical hypothyroidism in pregnant women with asymptomatic autoimmune thyroid disorders," Journal of Clinical Endocrinology and Metabolism, vol. 79, no. 1, pp. 197-204, 1994.

[39] J. H. Lazarus and S. Othman, "Thyroid disease in relation to pregnancy," Clinical Endocrinology, vol. 34, no. 1, pp. 91-98, 1991.

[40] J. H. Lazarus, "Prediction of postpartum thyroiditis," European Journal of Endocrinology, vol. 139, no. 1, pp. 12-13, 1998.

[41] A. P. Weetman, "Prediction of post-partum thyroiditis," Clinical Endocrinology, vol. 41, no. 1, pp. 7-8, 1994.

[42] A. Stagnaro-Green, S. H. Roman, R. H. Cobin, E. El-Harazy, A. Alvarez-Marfany, and T. F. Davies, "Detection of at-risk pregnancy by means of highly sensitive assays for thyroid autoantibodies," Journal of the American Medical Association, vol. 264, no. 11, pp. 1422-1425, 1990.

[43] D. Glinoer, M. F. Soto, P. Bourdoux et al., "Pregnancy in patients with mild thyroid abnormalities: maternal and neonatal repercussions," Journal of Clinical Endocrinology and Metabolism, vol. 73, no. 2, pp. 421-427, 1991.

[44] B. Lejeune, J. P. Grun, P. De Nayer, G. Servais, and D. Glinoer, "Antithyroid antibodies underlying thyroid abnormalities and miscarriage or pregnancy induced hypertension," British Journal of Obstetrics and Gynaecology, vol. 100, no. 7, pp. 669-672, 1993.

[45] T. Iijima, H. Tada, Y. Hidaka, N. Mitsuda, Y. Murata, and N. Amino, "Effects of autoantibodies on the course of pregnancy and fetal growth," Obstetrics and Gynecology, vol. 90, no. 3, pp. 364-369, 1997.
[46] T. Bagis, A. Gokcel, and E. S. Saygili, "Autoimmune thyroid disease in pregnancy and the postpartum period: relationship to spontaneous abortion," Thyroid, vol. 11, no. 11, pp. 1049-1053, 2001.

[47] F. Ghafoor, M. Mansoor, T. Malik et al., "Role of thyroid peroxidase antibodies in the outcome of pregnancy," Journal of the College of Physicians and Surgeons Pakistan, vol. 16, no. 7, pp. 468-471, 2006.

[48] R. Negro, G. Formoso, T. Mangieri, A. Pezzarossa, D. Dazzi, and H. Hassan, "Levothyroxine treatment in euthyroid pregnant women with autoimmune thyroid disease: effects on obstetrical complications," Journal of Clinical Endocrinology and Metabolism, vol. 91, no. 7, pp. 2587-2591, 2006.

[49] D. E. Pratt, G. Kaberlein, A. Dudkiewicz, V. Karande, and N. Gleicher, "The association of antithyroid antibodies in euthyroid nonpregnant women with recurrent first trimester abortions in the next pregnancy," Fertility and Sterility, vol. 60, no. 6, pp. 1001-1005, 1993.

[50] D. Pratt, M. Novotny, G. Kaberlein, A. Dudkiewicz, and N. Gleicher, "Antithyroid antibodies and the association with non-organ-specific antibodies in recurrent pregnancy loss," American Journal of Obstetrics and Gynecology, vol. 168, no. 3, pp. 837-841, 1993.

[51] S. Bussen and T. Steck, "Thyroid autoantibodies in euthyroid non-pregnant women with recurrent spontaneous abortions," Human Reproduction, vol. 10, no. 11, pp. 2938-2940, 1995.

[52] S. S. Bussen and T. Steck, "Thyroid antibodies and their relation to antithrombin antibodies, anticardiolipin antibodies and lupus anticoagulant in women with recurrent spontaneous abortions (antithyroid, anticardiolipin and antithrombin autoantibodies and Lupus anticoagulant in habitual aborters)," European Journal of Obstetrics Gynecology and Reproductive Biology, vol. 74, no. 2, pp. 139-143, 1997.

[53] W. H. Kutteh, D. L. Yetman, A. C. Carr, L. A. Beck, and R. T. Scott, "Increased prevalence of antithyroid antibodies identified in women with recurrent pregnancy loss but not in women undergoing assisted reproduction," Fertility and Sterility, vol. 71, no. 5, pp. 843-848, 1999.

[54] Y. Shoenfeld, H. J. A. Carp, V. Molina et al., "Autoantibodies and prediction of reproductive failure," American Journal of Reproductive Immunology, vol. 56, no. 5-6, pp. 337-344, 2006.

[55] A. T. Iravani, M. M. Saeedi, J. Pakravesh, S. Hamidi, and M. Abbasi, "Thyroid autoimmunity and recurrent spontaneous abortion in Iran: a case-control study," Endocrine Practice, vol. 14, no. 4, pp. 458-464, 2008.

[56] A. Stagnaro-Green, "Maternal thyroid disease and preterm delivery," Journal of Clinical Endocrinology and Metabolism, vol. 94, no. 1, pp. 21-25, 2009.

[57] R. Negro, "Thyroid autoimmunity and pre-term delivery: brief review and meta-analysis," Journal of Endocrinological Investigation, vol. 34, no. 2, pp. 155-158, 2011.

[58] Birmingham Clinical Trials Unit, "Randomised Controlled Trial of the Efficacy and Mechanism of Levothyroxine Treatment on Pregnancy and Neonatal Outcomes in Women with Thyroid Antibodies (TABLET)," In, http://www.birmingham.ac.uk, University of Birmingham (UK), EudraCT Number: 20011-000719-19, ISRCTN Number: 15948785, http://www .birmingham.ac.uk/research/activity/mds/trials/bctu/trials/ womens/tablet/index.aspx.

[59] B. M. Casey, J. S. Dashe, C. Y. Spong, D. D. McIntire, K. J. Leveno, and G. F. Cunningham, "Perinatal significance of isolated maternal hypothyroxinemia identified in the first half 
of pregnancy," Obstetrics and Gynecology, vol. 109, no. 5, pp. 1129-1135, 2007.

[60] V. J. Pop, E. P. Brouwers, H. L. Vader, T. Vulsma, A. L. van Baar, and J. J. de Vijlder, "Maternal hypothyroxinaemia during early pregnancy and subsequent child development: a 3-year followup study," Clinical Endocrinology, vol. 59, no. 3, pp. 282-288, 2003.

[61] J. Henrichs, J. J. Bongers-Schokking, J. J. Schenk et al., "Maternal thyroid function during early pregnancy and cognitive functioning in early childhood: the Generation R study," Journal of Clinical Endocrinology and Metabolism, vol. 95, no. 9, pp. 42274234, 2010.

[62] Y. Li, Z. Shan, W. Teng et al., "Abnormalities of maternal thyroid function during pregnancy affect neuropsychological development of their children at 25-30 months," Clinical Endocrinology, vol. 72, no. 6, pp. 825-829, 2010.

[63] E. Oken, L. E. Braverman, D. Platek, M. L. Mitchell, S. L. Lee, and E. N. Pearce, "Neonatal thyroxine, maternal thyroid function, and child cognition," Journal of Clinical Endocrinology and Metabolism, vol. 94, no. 2, pp. 497-503, 2009.

[64] J. Chevrier, K. G. Harley, K. Kogut, N. Holland, C. Johnson, and B. Eskenazi, "Maternal thyroid function during the second half ofpregnancy and child neurodevelopment at 6,12, 24, and 60 months of age," Journal of Thyroid Research, vol. 2011, Article ID 426427, 13 pages, 2011.

[65] J. Julvez, M. Alvarez-Pedrerol, M. Rebagliato et al., “Thyroxine levels during pregnancy in healthy women and early child neurodevelopment," Epidemiology, vol. 24, no. 1, pp. 150-157, 2013.

[66] G. J. Canaris, N. R. Manowitz, G. Mayor, and E. C. Ridgway, "The Colorado thyroid disease prevalence study," Archives of Internal Medicine, vol. 160, no. 4, pp. 526-534, 2000.

[67] M. Abalovich, N. Amino, L. A. Barbour et al., "Clinical practice guideline: management of thyroid dysfunction during pregnancy and postpartum: an endocrine society clinical practice guideline," Journal of Clinical Endocrinology and Metabolism, vol. 92, no. 8, pp. S1-S47, 2007.

[68] B. Vaidya, S. Anthony, M. Bilous et al., "Brief report: detection of thyroid dysfunction in early pregnancy: universal screening or targeted high-risk case finding?" Journal of Clinical Endocrinology and Metabolism, vol. 92, no. 1, pp. 203-207, 2007.

[69] J. Horacek, S. Spitalnikova, B. Dlabalova et al., "Universal screening detects two-times more thyroid disorders in early pregnancy than targeted high-risk case finding," European Journal of Endocrinology, vol. 163, no. 4, pp. 645-650, 2010.

[70] D. L. Chang, A. M. Leung, L. E. Braverman, and E. N. Pearce, "Thyroid testing during pregnancy at an Academic Boston area medical center," Journal of Clinical Endocrinology and Metabolism, vol. 96, no. 9, pp. E1452-E1456, 2011.

[71] J. E. Haddow, M. R. McClain, G. E. Palomaki, E. M. Kloza, and J. Williams, "Screening for thyroid disorders during pregnancy: results of a survey in Maine," American Journal of Obstetrics and Gynecology, vol. 194, no. 2, pp. 471-474, 2006.

[72] M. R. Haymart, "The role of clinical guidelines in patient care: thyroid hormone replacement in women of reproductive age," Thyroid, vol. 20, no. 3, pp. 301-307, 2010.

[73] A. J. Blatt/surname, J. M. Nakamoto, and H. W. Kaufman, "National status of testing for hypothyroidism during pregnancy and postpartum," Journal of Clinical Endocrinology and Metabolism, vol. 97, no. 3, pp. 777-784, 2012.

[74] B. Vaidya, A. Hubalewska-Dydejczyk, P. Laurberg, R. Negro, F. Vermiglio, and K. Poppe, "Treatment and screening of hypothyroidism in pregnancy: results of a European survey," European Journal of Endocrinology, vol. 166, no. 1, pp. 49-54, 2012.

[75] S. F. Thung, E. F. Funai, and W. A. Grobman, "The costeffectiveness of universal screening in pregnancy for subclinical hypothyroidism," American Journal of Obstetrics and Gynecology, vol. 200, no. 3, pp. 267.el-267.e7, 2009.

[76] C. Dosiou, J. Barnes, A. Schwartz, R. Negro, L. Crapo, and A. Stagnaro-Green, "Cost-effectiveness of universal and risk-based screening for autoimmune thyroid disease in pregnant women," Journal of Clinical Endocrinology and Metabolism, vol. 97, no. 5, pp. 1536-1546, 2012. 


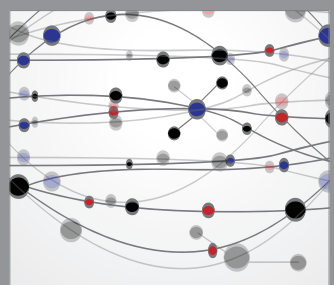

The Scientific World Journal
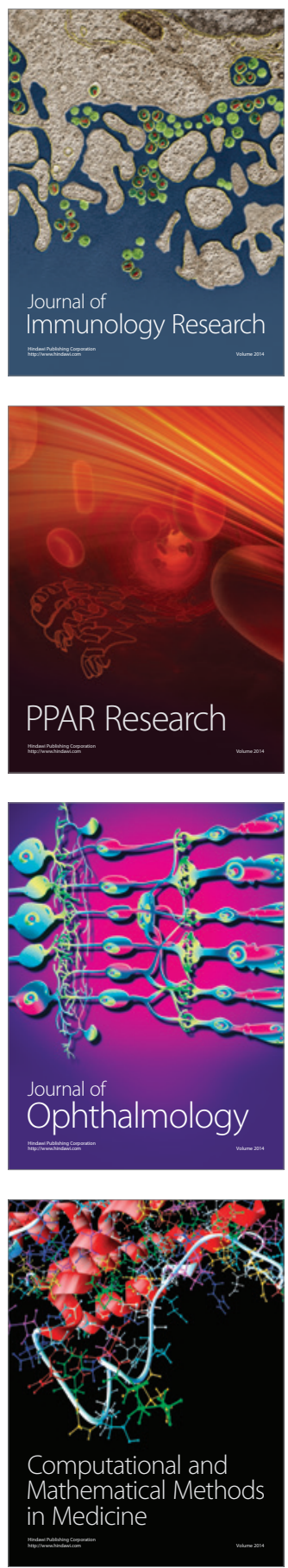

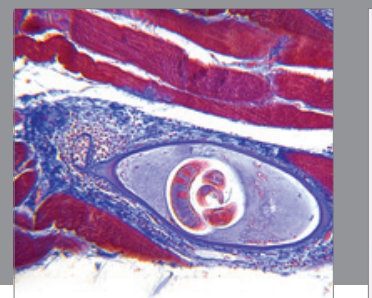

Gastroenterology

Research and Practice
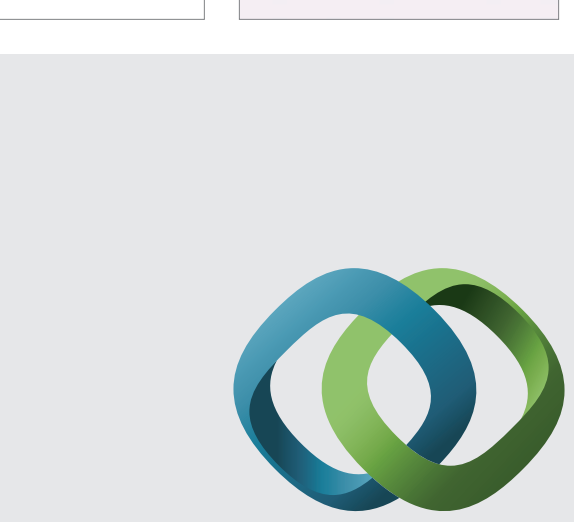

\section{Hindawi}

Submit your manuscripts at

http://www.hindawi.com
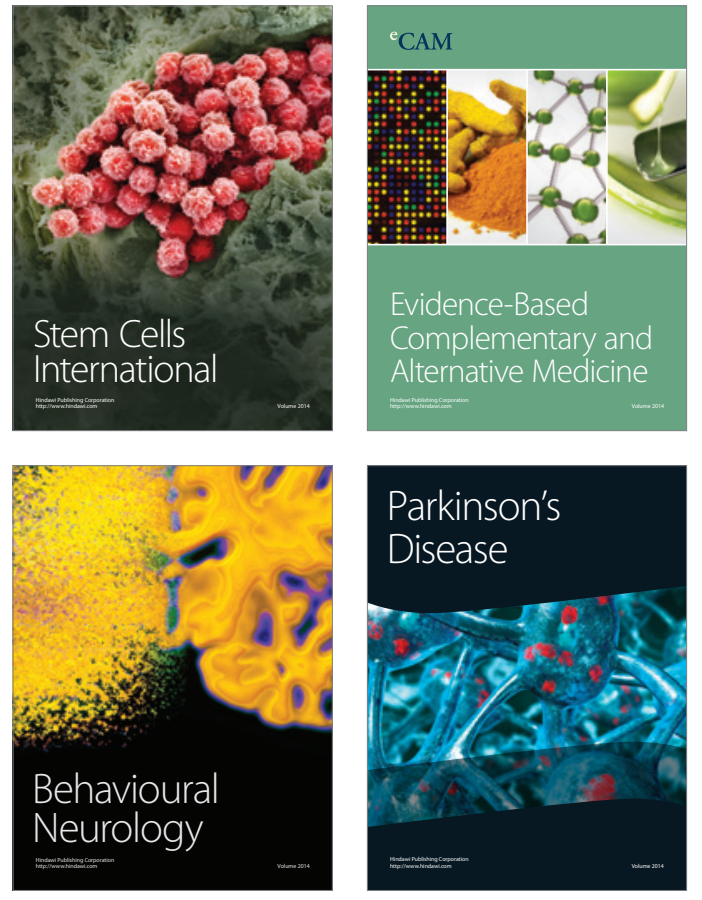
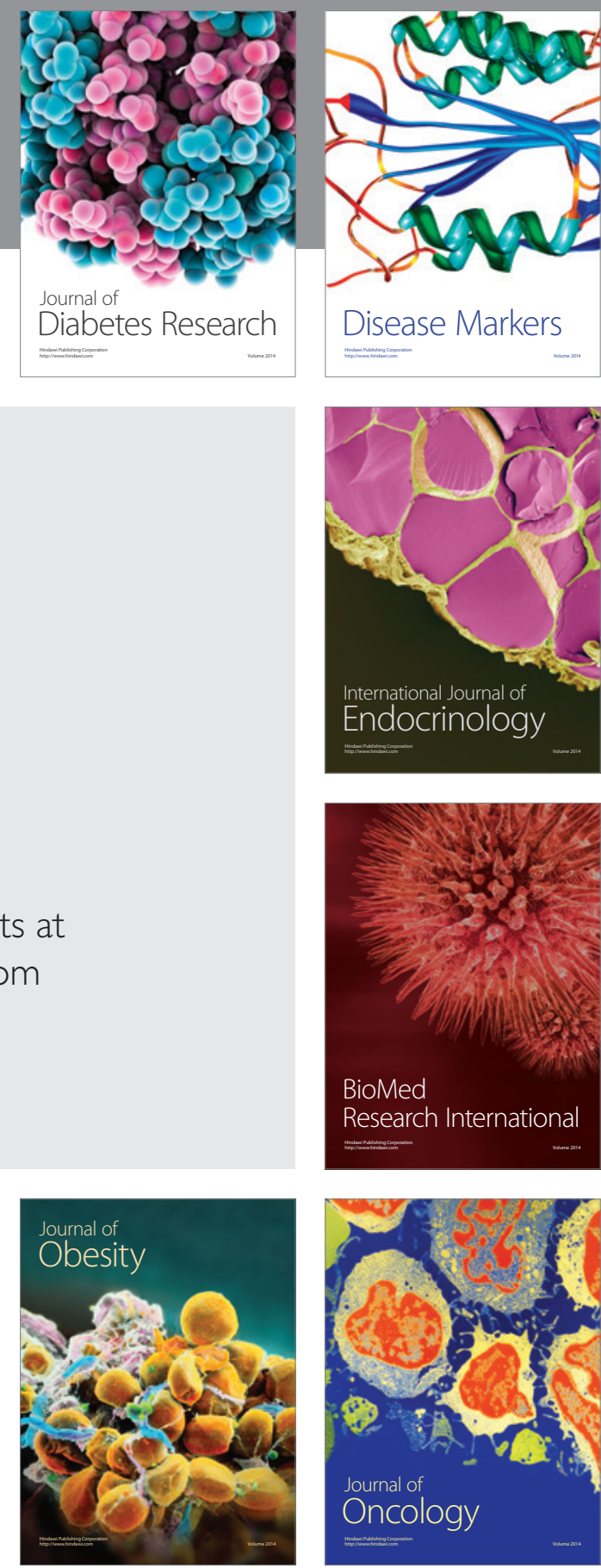

Disease Markers
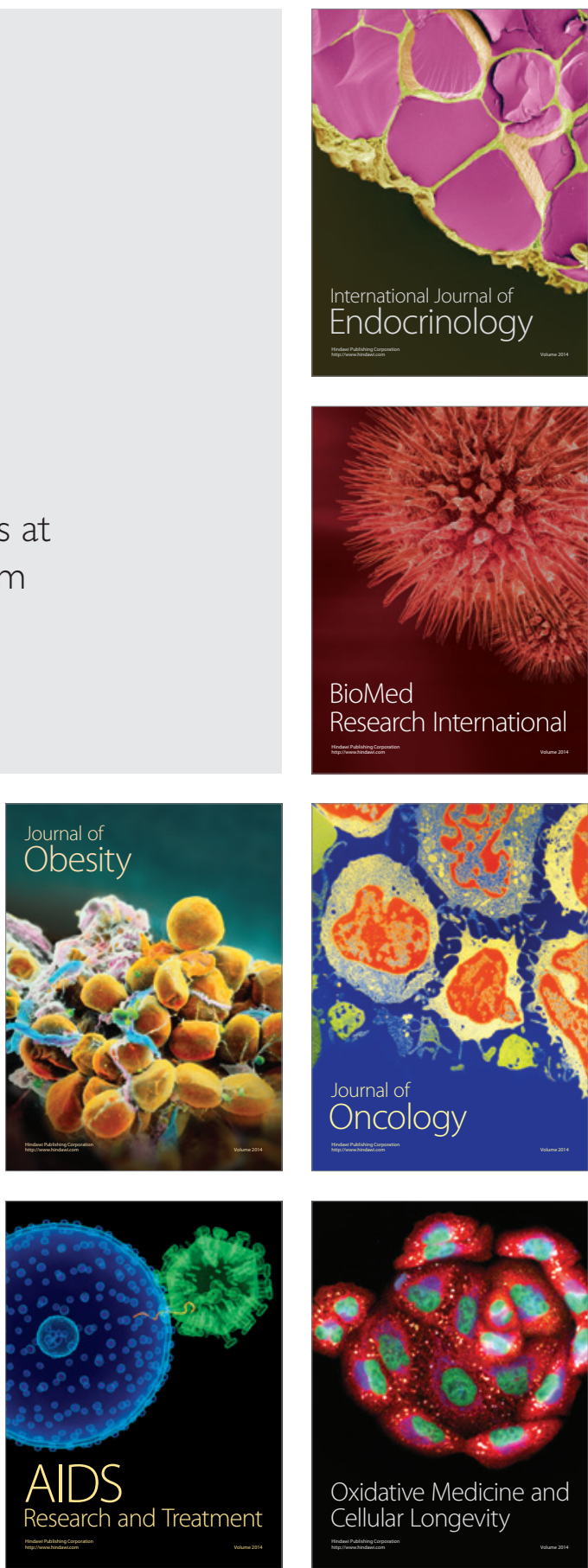